\title{
Influencia de la cultura en el desarrollo de la independencia funcional
}

\author{
Montserrat Santamaría-Vázquez, Valeriana Guijo-Blanco \\ Facultad de Ciencias de la Salud, Universidad de Burgos, Burgos, España
}

\begin{abstract}
Resumen: Introducción: Los aspectos culturales son considerados desde la disciplina de la Terapia Ocupacional como claves para entender el desempeño y el desarrollo. Objetivo: comparar el desempeño ocupacional entre niños sano con edades entre 3 y 7 años, en las actividades de la vida diaria entre diferentes países como son España, Noruega y Taiwán. Métodos: Se trata de un estudio comparativo entre España, Noruega y Taiwán. Ha utilizado la escala de habilidades funcionales del Pediatric Evaluation Disability Inventory (PEDI). Posteriormente se han comparado los datos de los tres mediante el uso de la prueba t no pareada de Welch. Resultados: Los resultados muestran diferencias significativas entre las tres muestras en todos los dominios de las escalas funcionales del PEDI y para todos los tramos de edad. Los niños taiwaneses puntúan más que los niños españoles y noruegos, y los españoles más que los noruegos en todos los dominios del PEDI. Conclusion: La cultura influye de forma determinante en los patrones de desarrollo en las habilidades relativas a la adquisición de la independencia en las AVDs. Antes del desarrollo de una intervención, el terapeuta ocupacional deber tener en cuenta las diferentes cuestiones relacionadas con la cultura.
\end{abstract}

Palabras clave: Independencia, Cultura, Desarrollo infantil, Actividades de la vida diaria.

\section{Influência da cultura no desenvolvimento da independência funcional}

Resumo: Introdução: Aspectos culturais são considerados a partir da disciplina de terapia ocupacional como chaves para a compreensão do desempenho e do desenvolvimento. Objetivo: comparar o desempenho ocupacional entre as crianças saudáveis, com idades entre 3 e 7 anos, nas realização de atividades da vida diária (AVD) entre diferentes países. Método: Estudo comparativo a partir de dados da Espanha, Noruega e Taiwan. Foi utilizada a escala de habilidades funcionais da Pediatric Evaluation Disability Inventory (PEDI). Foi feita a comparação dos dados dos três países, utilizando o teste não pareado Welch. Resultados: Os resultados mostram diferenças significativas entre as três amostras em todos os domínios de escalas funcionais de PEDI e para todas as faixas etárias. Crianças de Taiwan pontuaram mais do que as crianças espanholas e norueguesas, e crianças espanholas tiveram score mais elevado do que os noruegueses em todos os domínios funcionais do PEDI. Conclusão: A cultura está muito ligada aos padrões de desenvolvimento em habilidades para a aquisição de independência em AVD. Antes do planejamento de uma intervenção, o terapeuta ocupacional deve levar em conta diferentes questões relacionadas com a cultura.

Palavras-chave: Independência, Cultura, Desenvolvimento Infantil, Atividades de Vida Diária.

\section{Cultural influence in the development of functional independence}

\begin{abstract}
Introduction: The occupational therapy discipline considers cultural aspects as keys to understanding the performance and development. Objective: To compare occupational performance among healthy children aged 3 and 7 years in daily life activities in different countries. Method: This is a comparative study, from Spain, Norway and Taiwan. We used the Pediatric Evaluation of Disability Inventory (PEDI) scale of functional skills. Data from the three countries were compared using the Welch unpaired test. Results: The results show that there are significant
\end{abstract}

Autor para la correspondencia: Montserrat Santamaría-Vázquez, Facultad de Ciencias de la Salud, Universidad de Burgos, Paseo Comendadores, s/n, 09001, Burgos, España, e-mail: msvazquez@ubu.es

Recibido Abr. 14, 2015; $1^{\text {a }}$ Revisión Oct. 27, 2015; 2ª Revisión Dic. 09, 2015; 3ª Revisión Marzo 18, 2016; Aceptado Mayo 09, 2016. 
differences between the three samples in all domains of the PEDI functional scales and for all age groups. Taiwanese children scored higher than Spanish and Norwegian children; and Spanish children scored higher than Norwegians in all of the PEDI functional domains. Conclusion: Culture has a decisive role in the development patterns of skills for acquiring independence in ADLs. Before planning an intervention, occupational therapist should take into account different cultural related issues.

Keywords: Independence, Culture, Child Development, Activities of Daily Living.

\section{Introducción}

El ejercicio de la terapia ocupacional puede definirse como una danza cuidadosamente orquestada de las propiedades dinámicas de los ambientes humanos y no humanos que brindan significado y propósito a la vida de una persona (MASAGATANI, 1998, p. 147).

La cultura orienta e impulsa las actividades de los miembros de una determinada sociedad (FLORES MARTOS, 2006) convirtiéndose en una dimensión clave para analizar el comportamiento ocupacional que es el objeto de la Terapia Ocupacional (TO). La cultura no define simplemente lo que una persona decide hacer, sino también el significado y la interpretación de las acciones propias y ajenas (MATTINGLY; BEER, 1998). Por eso los estudios sobre la influencia de la cultura son necesarios para entender las conductas de la persona a lo largo de su ciclo vital.

El Modelo Persona, Entorno y Desempeño Ocupacional (Person, Environment and Occupational Performance) (LAW et al., 1996) estima que la capacidad para llevar a cabo el desempeńo ocupacional está influenciada por aspectos personales -como son las funciones y estructuras corporales, las creencias y valores-, por aspectos propios de la ocupación -tales como los objetos y sus características, las demandas sociales, el ritmo-y por el entorno -que engloba los contextos socioeconómico, cultural, físico y social-. Desde este modelo se entiende que el entorno procura el contexto para que la persona lleve a cabo su propio desempeño ocupacional, y que ambos, desempeño y entorno, se influyen mutuamente. Los entornos en los que se situa la persona no son estáticos, sino que están en continuo cambio, lo que implica que los comportamientos o conductas que se muestran a través del desempeño, también cambian (RIGBY; LETTS, 2003) y ese cambio ha de ser observable.

La influencia del entorno le llega al niño a través del microsistema (en términos de Bronfenbrenner), es decir, a través de las creencias, los patrones de conducta, los valores, los estilos de vida, etc. que conforman el macrosistema (CÓRDOBA IÑESTA, 2008). La cultura marca, así, las relaciones entre los adultos y los niños pautando los procesos psicosociales de la crianza es decir, las pautas, las prácticas y las creencias acerca de la crianza (PARMAR; SUPER, 2004; IZZEDIN; PACHAJOA, 2009; CHUA, 2011). Cómo los padres favorecen la independencia, se ocupan de la enseñanza de habilidades o ejercen la disciplina son elementos claves que condicionan el desempeño del niño. Si la elección de los padres puede variar en función de un contexto cultural determinado, cabe esperar que se observen diferencias en el logro de capacidades por el niño en su desempeño. Así, Case-Smith y Clifford O'Brien (2010) reflexionan sobre lo determinante que resulta en las ocupaciones infantiles las ideas de interdependencia o de autonomía, y ejemplifican diferencias entre culturas que ilustran como afectan a actividades diarias tales como dormir; igualmente Adair et al. (2004) encuentran diferencias culturales en las creencias sobre los hábitos de higiene. Así mismo, los modelos y pautas culturales de crianza y las metas del desarrollo que los padres proponen para sus hijos operan en un contexto de multidimensional que potencia o dificulta el desempeño ocupacional del niño. Estos factores incluyen la biología, el medio físico, la estructura familiar, el trabajo parental, las relaciones entre grupos, y la economía de la sociedad (GREENFIELD; SUZUKI, 1998).

El presente estudio se plantea como objetivo comparar los datos de diferentes muestras, una española, una noruega y otra taiwanesa, con el fin de comprobar la existencia o no de diferencias en el desempeño ocupacional en las actividades de la vida diaria de niños de corta edad que viven en diferentes contextos culturales, partiendo de la hipótesis de-que el desempeño de los tres grupos es diferente puesto que las diferencias culturales son importantes.

\section{Método}

\subsection{Diseño y participantes}

Se trata de un estudio comparativo (meta-análisis) entre los resultados de tres estudios realizados en España, Noruega y Taiwán. Se trata de una muestra de 530 niños sanos entre 3 y 7 años, sin ningún tipo 
de alteración del desarrollo, sobre los que se recogieron datos sobre la independencia en las actividades de la vida diaria a través del PEDI. Los criterios de inclusión y exclusión de cada una de las submuestras son descritos por los respectivos autores y publicados en los correspondientes trabajos (BERG et al., 2008; CHEN et al., 2010; SANTAMARÍA VÁZQUEZ, 2014), siendo que todos coinciden en que los nińos no tengan ningun diagnóstico que afecte al desarrollo y cumplan los criterios de edad.

Los datos demográficos de cada una de las muestras noruega, taiwanesa y española, son igualmente los especificados en los anteriores trabajos, no habiendo tenido acceso a las bases de datos de los trabajos noruego y taiwanés. En la Tabla 1, se especifica el tamaño muestral de cada estudio dividido por tramo de edad.

\subsection{Instrumento}

Para valorar el desempeño funcional en las actividades de la vida diaria (AVDs) de los niños, se ha utilizado la escala de habilidades funcionales del Pediatric Evaluation Disability Inventory (PEDI) (HALEY et al., 1992). Esta escala está divida en tres dominios: cuidado personal, movilidad y función social. El dominio de cuidado personal contiene 73 ítems que recogen información sobre la capacidad del nińo para actividades tales como comer, vestirse, peinarse o lavarse los dientes; el dominio de movilidad reúne 59 ítems dónde se constata por ejemplo, si el niño es capaz o no de caminar, de transportar objetos o de subir y bajar escaleras; finalmente el dominio de función social con 65 ítems, se centra en la capacidad de comprensión de frases y palabras, las interacciones durante el juego, la colaboración en las tareas del hogar o el funcionamiento dentro de la comunidad entre otras.

Todos los dominios se puntúan a través de una escala dicotómica, dónde se califica como 1 cuando el niño es capaz de realizar la tarea descrita en cada uno de los ítems sin ayuda, y como 0 cuando el niño no es capaz de realizarla o necesita ayuda. Se obtiene por un lado, una puntuación para cada uno de los dominios resultado del sumatorio de aquellos ítems dónde se le ha identificado al nińo como capaz, y una puntuación global de la escala, suma de las puntuaciones de los tres dominios.

El PEDI ha sido traducido al noruego, taiwanés y español, y sus propiedades psicométricas han sido analizadas por diferentes autores, obteniendo unas adecuadas propiedades psicométricas en todos los casos (BERG et al., 2004; CHENG et al., 2009; GARCÍA BÁSCONES, 2013).

\subsection{Procedimiento}

Tras la recopilación de los datos de las tres publicaciones, se realizan análisis estadísticos y se analizan resultados, teniendo en cuenta las siguientes variables de estudio:

- Cuidado Personal. Esta variable se define como la media de puntuación obtenida en el dominio de cuidado personal de las escalas de habilidades funcionales del PEDI. Se establece esta variable por tramo de edad.

- Movilidad. Esta variable se define como la media de puntuación obtenida en el dominio de movilidad correspondiente a las escalas de habilidades funcionales del PEDI. Se establece esta variable por tramo de edad.

- Función social. Esta variable se define como la media de puntuación obtenida en el dominio de función social dentro de las escalas de habilidades funcionales del PEDI. Se establece esta variable por tramo de edad.

- Cuidado personal DT. Esa variable es el resultado de la desviación típica obtenida en el dominio de cuidado personal de las escalas de habilidades funcionales del PEDI. Se establece esta variable por tramo de edad.

- Movilidad DT. Esa variable es el resultado de la desviación típica obtenida en el dominio de movilidad de las escalas de habilidades funcionales del PEDI. Se establece esta variable por tramo de edad.

- Función social DT. Esa variable es el resultado de la desviación típica obtenida en el dominio de función social de las escalas de habilidades funcionales del PEDI. Se establece esta variable por tramo de edad.

Tabla 1. Número del tamaño muestral, según país y según tramo de edad.

\begin{tabular}{|c|c|c|c|c|c|c|c|c|}
\hline & \multicolumn{7}{|c|}{ Tramos de edad (aa/mm/dd) } & \multirow[b]{2}{*}{ Total } \\
\hline & $\begin{array}{c}3 / 0 / 0 \\
3 / 5 / 30\end{array}$ & $\begin{array}{c}3 / 6 / 0 \\
3 / 11 / 30\end{array}$ & $\begin{array}{c}4 / 0 / 0 \\
4 / 5 / 30\end{array}$ & $\begin{array}{c}4 / 6 / 0 \\
4 / 11 / 30\end{array}$ & $\begin{array}{c}5 / 0 / 0 \\
5 / 5 / 30\end{array}$ & $\begin{array}{c}5 / 6 / 0 \\
5 / 11 / 30\end{array}$ & $\begin{array}{l}6 / 0 / 0 \\
6 / 5 / 30\end{array}$ & \\
\hline España n & 17 & 34 & 40 & 47 & 25 & 31 & 5 & 199 \\
\hline Noruega $n$ & 16 & 18 & 18 & 18 & 24 & 22 & & 116 \\
\hline Taiwán n & 38 & 34 & 37 & 35 & 30 & 38 & 35 & 215 \\
\hline
\end{tabular}


Los tres estudios informan de que se han respetado todos los principios éticos y se han seguido las normas relativas a la protección de datos.

\subsection{Análisis estadísticos}

Se han tomado datos de medias y desviaciones típicas por los rangos de edad establecidos en el manual del PEDI (HALEY et al., 1992) de las tres submuestras (descritas y especificadas en cada uno de los tres documentos), y posteriormente se han comparado estos resultados con las tres submuestras entre sí.

Para determinar si había o no diferencias significativas entre las muestras, se ha utilizado la prueba t no pareada de Welch, la cual asume la distribución normal de la población, varianzas desconocidas y diferentes, puesto que únicamente se ha tenido acceso al tamaño muestral y a los datos de medias y desviación típicas de las muestras noruega y taiwanesa. La prueba t ha sido realizada con el software libre GraphPad, disponible en internet.

\section{Resultados}

Los datos relativos a las puntuaciones obtenidas en cada dominio por las tres muestras se presentan en las Tablas 2, 3 y 4. Los datos mostrados sobre Noruega y Taiwán son los obtenidos por Berg y Chen respectivamente (BERG et al., 2008; CHEN et al., 2010). Estos datos han servido para la comparación

Tabla 2. Estadísticos descriptivos de las tres muestras en el dominio de Cuidado Personal de la escala de habilidades funcionales del PEDI (HALEY et al., 1992).

\begin{tabular}{|c|c|c|c|c|c|c|}
\hline \multirow{3}{*}{$\begin{array}{c}\text { Edad } \\
\mathrm{aa} / \mathrm{mm} / \mathrm{dd}\end{array}$} & \multicolumn{6}{|c|}{ Dominio de Cuidado Personal } \\
\hline & \multicolumn{2}{|c|}{ Noruega } & \multicolumn{2}{|c|}{ Taiwán } & \multicolumn{2}{|c|}{ España } \\
\hline & Media & DT & Media & DT & Media & DT \\
\hline $\begin{array}{c}3 / 0 / 0 \\
3 / 5 / 30\end{array}$ & 40,4 & 6,7 & 64,3 & 7,9 & 56,9 & 7,55 \\
\hline $\begin{array}{c}3 / 6 / 0 \\
3 / 11 / 30\end{array}$ & 37,6 & 8,1 & 67,3 & 4,9 & 58,02 & 5,82 \\
\hline $\begin{array}{c}4 / 0 / 0 \\
4 / 5 / 30\end{array}$ & 36,7 & 6,1 & 74,0 & 5,6 & 61,9 & 5,17 \\
\hline $\begin{array}{c}4 / 6 / 0 \\
4 / 11 / 30\end{array}$ & 37,9 & 11,0 & 74,5 & 6,2 & 65,21 & 3,57 \\
\hline $\begin{array}{c}5 / 0 / 0 \\
5 / 5 / 30\end{array}$ & 40,3 & 5,4 & 78,3 & 7,0 & 64,6 & 3,5 \\
\hline $\begin{array}{c}5 / 6 / 0 \\
5 / 11 / 30\end{array}$ & 39,2 & 5,4 & 78,4 & 10,6 & 66,29 & 3,48 \\
\hline $\begin{array}{c}6 / 0 / 0 \\
6 / 5 / 30\end{array}$ & & & 82,6 & 9,0 & 69,6 & 4,15 \\
\hline
\end{tabular}

Tabla 3. Estadísticos descriptivos de las tres muestras en el dominio de Movilidad de la escala de habilidades funcionales del PEDI (HALEY et al., 1992).

\begin{tabular}{|c|c|c|c|c|c|c|}
\hline \multirow{3}{*}{$\begin{array}{c}\text { Edad } \\
\mathrm{aa} / \mathrm{mm} / \mathrm{dd}\end{array}$} & \multicolumn{6}{|c|}{ Dominio de Movilidad } \\
\hline & \multicolumn{2}{|c|}{ Noruega } & \multicolumn{2}{|c|}{ Taiwán } & \multicolumn{2}{|c|}{ España } \\
\hline & Media & DT & Media & DT & Media & DT \\
\hline $\begin{array}{c}3 / 0 / 0 \\
3 / 5 / 30\end{array}$ & 40,8 & 5,0 & 82,4 & 6,3 & 54,29 & 1,49 \\
\hline $\begin{array}{c}3 / 6 / 0 \\
3 / 11 / 30\end{array}$ & 41,2 & 4,5 & 84,0 & 8,1 & 53,8 & 1,48 \\
\hline $\begin{array}{c}4 / 0 / 0 \\
4 / 5 / 30\end{array}$ & 40,4 & 6,4 & 86,8 & 6,0 & 54,9 & 1,54 \\
\hline $\begin{array}{c}4 / 6 / 0 \\
4 / 11 / 30\end{array}$ & 38,5 & 10,5 & 90,7 & 6,9 & 55,48 & 1,26 \\
\hline $\begin{array}{c}5 / 0 / 0 \\
5 / 5 / 30\end{array}$ & 49,8 & 9,1 & 93,6 & 6,7 & 56,5 & 1,44 \\
\hline $\begin{array}{c}5 / 6 / 0 \\
5 / 11 / 30\end{array}$ & 41,3 & 12,5 & 95,7 & 4,8 & 56,48 & 1,43 \\
\hline $\begin{array}{c}6 / 0 / 0 \\
6 / 5 / 30\end{array}$ & & & 97,2 & 4,3 & 56,6 & 1,14 \\
\hline
\end{tabular}


con los resultados entre los tres países, a través del cálculo de la prueba t de Welch.

En la Figura 1 se representan las medias obtenidas por tramo de edad en los diferentes dominios de la escala de habilidades funcionales del PEDI.

Los resultados del cálculo de la prueba t de Welch, muestran diferencias estadísticamente significativas en la comparación entre las muestras española, noruega y taiwanesa (véase Tabla 5, 6 y 7) para las habilidades funcionales. El sentido de estas diferencias se puede apreciar en la Figura 1; siendo que los nińos taiwaneses puntúan más alto en todos los dominios que los españoles y estos más que los niños noruegos. Las curvas siguen trayectorias diferentes debido a que la magnitud de las diferencias no es constante en el tramo de edades estudiados, no obstante, el logro de los niños taiwaneses es mayor que el de los españoles y los noruegos.

\section{Discusión}

Los resultados de este estudio ponen de manifiesto que la cultura influye directamente en el desarrollo de la independencia en las actividades de la vida diaria. Incluso en países europeos como pudieran ser España y Noruega. Abordar la razón de estas diferencias es complejo debido a la cantidad de factores que conforman el ambiente y la cultura.

Las diferentes políticas en términos de crianza y conciliación de la vida familiar, pueden ser causa de estos desajustes. Los países nórdicos son considerados como los mejores países del mundo para criar un hijo según diferentes informes (SAVE THE CHILDREN, 2013, 2014) estableciendo como uno de los criterios, la generosidad de la políticas sociales respecto a la maternidad, que incluyen prestaciones a cambio de cuidar de los hijos. Puede que la presencia de la madre en casa sea una de las causas para adquirir los hábitos de independencia en las AVDs de forma más tardía, pues en este sentido, los investigadores taiwaneses argumentan como razón a sus resultados, la incorporación de la mujer taiwanesa al mundo laboral (CHEN et al., 2010).

Otro factor a tener en cuenta, más allá de las políticas familiares, son los estilos de crianza. Los estilos de crianza occidentales y orientales son diferentes tal como muestra Amy Chua en su ensayo "Madres tigres, hijos leones" (2011). La cultura

Tabla 4. Estadísticos descriptivos de las tres muestras en el dominio de Función Social de la escala de habilidades funcionales del PEDI (HALEY et al., 1992).

\begin{tabular}{|c|c|c|c|c|c|c|}
\hline \multirow{3}{*}{$\frac{\text { Edad }}{\mathrm{aa} / \mathrm{mm} / \mathrm{dd}}$} & \multicolumn{6}{|c|}{ Dominio de Función Social } \\
\hline & \multicolumn{2}{|c|}{ Noruega } & \multicolumn{2}{|c|}{ Taiwán } & \multicolumn{2}{|c|}{ España } \\
\hline & Media & DT & Media & DT & Media & DT \\
\hline $\begin{array}{c}3 / 0 / 0 \\
3 / 5 / 30\end{array}$ & 47,3 & 4,6 & 61,4 & 5,1 & 50,70 & 3,67 \\
\hline $\begin{array}{c}3 / 6 / 0 \\
3 / 11 / 30\end{array}$ & 44,8 & 5,2 & 65,2 & 5,8 & 50,73 & 4,25 \\
\hline $\begin{array}{c}4 / 0 / 0 \\
4 / 5 / 30\end{array}$ & 43,7 & 6,5 & 66,6 & 4,9 & 54,35 & 3,74 \\
\hline $\begin{array}{c}4 / 6 / 0 \\
4 / 11 / 30\end{array}$ & 43,1 & 12,1 & 69,0 & 5,1 & 55,23 & 3,23 \\
\hline $\begin{array}{c}5 / 0 / 0 \\
5 / 5 / 30\end{array}$ & 45,0 & 6,1 & 74,8 & 8,1 & 57,16 & 2,67 \\
\hline $\begin{array}{c}5 / 6 / 0 \\
5 / 11 / 30\end{array}$ & 43,4 & 6,7 & 73,3 & 8,4 & 57,12 & 2,78 \\
\hline $\begin{array}{c}6 / 0 / 0 \\
6 / 5 / 30 \\
\end{array}$ & & & 77,9 & 9,0 & 56,8 & 3,76 \\
\hline
\end{tabular}
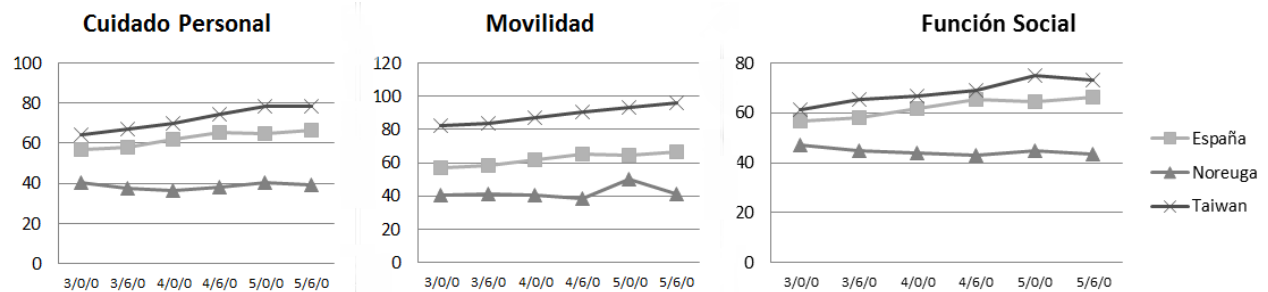

Figura 1. Representación gráfica de las puntuaciones obtenidas por las muestras española, noruega y taiwanesa. 
oriental parece ser más exigente y menos permisiva que las occidentales lo que guarda relación con la denominada "etnoteoría parental". Este concepto hace referencia precisamente a la influencia de la cultura en la forma de ejercer los roles paternos y maternos, y que por ejemplo, en relación al juego atribuye a los padres occidentales una mayor preocupación por este, puesto que ven en el juego un potencial de oportunidades para favorecer el desarrollo, mientras que los padres orientales se centran más en tareas

Tabla 5. Resultados de la prueba t de Welch, comparando la muestra española con la noruega.

\begin{tabular}{|c|c|c|c|c|c|c|c|c|c|c|c|c|}
\hline \multirow{2}{*}{$\frac{\text { Edad }}{\mathrm{aa} / \mathrm{mm} / \mathrm{dd}}$} & \multicolumn{4}{|c|}{ Dominio de Cuidado Personal } & \multicolumn{4}{|c|}{ Dominio de Movilidad } & \multicolumn{4}{|c|}{ Dominio de Función Social } \\
\hline & $\mathbf{t}$ & $\mathrm{p}$ & Diferencia & EE & $\mathrm{t}$ & $\mathrm{p}$ & Diferencia & EE & $\mathrm{t}$ & $\mathrm{p}$ & Diferencia & EE \\
\hline $\begin{array}{c}3 / 0 / 0 \\
3 / 5 / 30\end{array}$ & 6,64 & ,0001 & $16,5^{*}$ & 2,48 & 10,29 & ,0001 & 13,4 & 1,3 & 2,35 & ,0258 & 3,4 & 1,44 \\
\hline $\begin{array}{c}3 / 6 / 0 \\
3 / 11 / 30\end{array}$ & 9,48 & 0001 & 20,42 & 2,1 & 11,55 & 0001 & 12,60 & 1,09 & 4,13 & ,0003 & 5,9 & 1,42 \\
\hline $\begin{array}{c}4 / 0 / 0 \\
4 / 5 / 30\end{array}$ & 15,23 & ,0001 & 25,2 & 1,65 & 9,48 & ,0001 & 14,5 & 1,58 & 6,4 &, 0001 & 10,65 & 1,64 \\
\hline $\begin{array}{c}4 / 6 / 0 \\
4 / 11 / 30\end{array}$ & 10,32 & ,0001 & 27,30 & 2,64 & 6,80 & ,0001 & 16,90 & 2,48 & 4,19 & ,0006 & 12,13 & 2,89 \\
\hline $\begin{array}{c}5 / 0 / 0 \\
5 / 5 / 30\end{array}$ & 18,60 & ,0001 & 24,3 & 1,30 & 3,5 & ,0015 & 6,72 & 1,88 & 8,9 & ,0001 & 12,16 & 1,35 \\
\hline $\begin{array}{c}5 / 6 / 0 \\
5 / 11 / 30\end{array}$ & 20,6 & 0001 & 27 & 1,3 & 5,66 & ,0001 & 15,18 & 2,67 & 9,06 & ,0001 & 13,72 & 1,51 \\
\hline
\end{tabular}

*Que las diferencias de medias se expresen en positivo, significa que la población española puntúa más alto.

Tabla 6. Resultados de la prueba t de Welch, comparando la muestra española con la taiwanesa.

\begin{tabular}{|c|c|c|c|c|c|c|c|c|c|c|c|c|}
\hline \multirow{2}{*}{$\frac{\text { Edad }}{\mathrm{aa} / \mathrm{mm} / \mathrm{dd}}$} & \multicolumn{4}{|c|}{ Dominio de Cuidado Personal } & \multicolumn{4}{|c|}{ Dominio de Movilidad } & \multicolumn{4}{|c|}{ Dominio de Función Social } \\
\hline & $\mathrm{t}$ & $\mathrm{p}$ & Diferencia & $\mathrm{EE}$ & $\mathrm{t}$ & $\mathrm{p}$ & Diferencia & $\mathbf{E E}$ & $\mathrm{t}$ & $\mathrm{p}$ & Diferencia & EE \\
\hline $\begin{array}{c}3 / 0 / 0 \\
3 / 5 / 30\end{array}$ & 3,3 & 002 & & 223 & 5,36 & 0001 & & 1,08 & 8,89 & 0001 & & 1,203 \\
\hline $\begin{array}{r}3 / 6 / \\
3 / 11 /\end{array}$ & 7,12 &, 0 & $-9,28$ & 1,30 & 21,37 &, 0 & & 1,41 & 11,73 & 0001 & 7 & 1,23 \\
\hline & 7,2 & 0001 & 10 & 1,12 & 31,39 & 0001 & 2 & 1,01 & 12,25 & 0001 & $-12,25$ & ,999 \\
\hline & 7,9 &, 00 & 020 & 117 & 29,82 & 0001 & 2 & 1,18 & 14,01 & 0001 & 77 & 0,98 \\
\hline & 9,4 & ,0001 & $-13,7$ & 1,45 & 29,5 & ,0001 & $-37,08$ & 1,25 & 11,21 & ,0001 & 64 & 1,57 \\
\hline $\begin{array}{c}5 / 6 / 0 \\
5 / 11 / 30\end{array}$ & 6,6 & 0001 & $-12,11$ & 1,83 & 47,83 & 0001 & $-39,22$ & 0,82 & 11,14 & 0001 & $-16,18$ & 1,45 \\
\hline $\begin{array}{c}6 / 0 / 0 \\
6 / 5 / 30\end{array}$ & 5,4 &, 0003 & $-13,00$ & 2,4 & 45,73 & 0001 & $-38,76$ & 0,88 & 9,58 & ,0001 & $-21,1$ & 2,21 \\
\hline
\end{tabular}

*Que las diferencias de medias se expresen en negativo, significa que la población española puntúa más bajo.

Tabla 7. Resultados de la prueba t de Welch, comparando la muestra noruega con la taiwanesa.

\begin{tabular}{|c|c|c|c|c|c|c|c|c|c|c|c|c|}
\hline \multirow{2}{*}{$\begin{array}{c}\text { Edad } \\
\mathrm{aa} / \mathrm{mm} / \mathrm{dd}\end{array}$} & \multicolumn{4}{|c|}{ Dominio de Cuidado Personal } & \multicolumn{4}{|c|}{ Dominio de Movilidad } & \multicolumn{4}{|c|}{ Dominio de Función Social } \\
\hline & $\mathrm{t}$ & $\mathrm{p}$ & Diferencia & EE & $\mathrm{t}$ & $\mathrm{p}$ & Diferencia & EE & $\mathrm{t}$ & $\mathrm{p}$ & Diferencia & EE \\
\hline $\begin{array}{c}3 / 0 / 0 \\
3 / 5 / 30\end{array}$ & 10,5 & ,0001 & $-23,9^{*}$ & 2,25 & 23,44 & ,0001 & $-41,6$ & 1,77 & 9,5 & 0001 & $-14,1$ & 1,47 \\
\hline $\begin{array}{c}3 / 6 / 0 \\
3 / 11 / 30\end{array}$ & 16,49 & ,0001 & $-29,7$ & 1,8 & 20,72 & ,0001 & $-42,8$ & 2,06 & 12,5 & ,0001 & $-20,4$ & 1,63 \\
\hline $\begin{array}{c}4 / 0 / 0 \\
4 / 5 / 30\end{array}$ & 22,5 & ,0001 & $-37,3$ & 1,65 & 26,33 & ,0001 & $-46,4$ & 1,76 & 14,58 & ,0001 & $-22,9$ & 1,57 \\
\hline $\begin{array}{c}4 / 6 / 0 \\
4 / 11 / 30\end{array}$ & 15,5 & ,0001 & $-36,6$ & 2,35 & 26,88 & ,0001 & $-52,2$ & 1,94 & 10,97 & ,0001 & $-25,9$ & 2,36 \\
\hline $\begin{array}{c}5 / 0 / 0 \\
5 / 5 / 30\end{array}$ & 21,8 & ,0001 & -38 & 1,73 & 20,36 & ,0001 & $-43,8$ & 2,15 & 14,9 & ,0001 & $-29,8$ & 1,99 \\
\hline $\begin{array}{c}5 / 6 / 0 \\
5 / 11 / 30\end{array}$ & 16,13 & ,0001 & $-39,2$ & 2,42 & 24,5 & ,0001 & $-54,4$ & 2,26 & 14,25 & ,0001 & $-29,9$ & 2,09 \\
\hline
\end{tabular}

*Que las diferencias de medias se expresen en negativo, significa que la población noruega puntúa más bajo. 
académicas (PARMAR; SUPER, 2004). En esta misma línea, Wise y da Silva (2007) argumentan que los padres otorgan a la independencia valores diferentes según la cultura de dónde provengan. En el caso concreto de los hábitos relacionados con el cuidado de los dientes, Adair et al. (2004) encontraron que había diferencias significativas en cuanto a las creencias relacionadas con el control que ejercían sobre sus hijos tanto en el número de cepillados al día como en la importancia de hacerlo, entre países como Bélgica y Noruega con China.

Gross-Loh (2013) realiza una revisión sobre la valoración de los padres en temas relacionados con la socialización, la participación en actividades fuera de casa o la independencia, mostrando por ejemplo que los padres de países como España y Noruega no dedican el mismo tiempo a ir con los niños a zonas de juego infantiles, o que en países de cultura oriental como Taiwán, los padres dan mucha importancia a las actividades grupales debido a la influencia de la cultura colectivista.

Otro de los argumentos a tener en cuenta para explicar estas diferencias es el relacionado con el conocimiento que tienen los padres a propósito del desarrollo de sus hijos. Son varios los estudios que ponen de manifiesto que cuanto mayor sea el conocimiento sobre el desarrollo, más fácil es acompañar a los hijos en este proceso y ayudarles a adquirir sus competencias (DICHTELMILLER et al., 1992; RIKHY et al., 2010; STEVENS, 1984). A propósito de España, y en relación al proceso de adquisición de la independencia en las AVDs, es incluso difícil encontrar textos científicos validados en el país que ofrezcan un criterio claro de a qué edad se considera adecuado la adquisición de las diferentes habilidades relativas a la autonomía. Por esta razón, no extraña que los padres espańoles adolezcan precisamente de un criterio ajustado de cuándo deben sus hijos realizar determinadas actividades, dificultado esto que puedan guiarles para conseguir esa mayor independencia.

Por otro lado es necesario abordar la cuestión del entorno físico, que tanto Law et al. (1996) como Rigby y Letts (2003) insisten en su papel como moldeador del desempeńo. Sobre este tema si bien no es posible ofrecer datos concretos, la observación de las de imágenes de la vida cotidiana (casas, parques, colegios, etc.) de los tres países estudiados, reflejan contextos físicos diferentes (tipos de camas, frecuencia en la presencia de escaleras dentro del hogar, etc.) que pueden haber influido en la base del desarrollo.

Sin duda son muchas cuestiones las que se pueden analizar, pero los resultados de este trabajo evidencian que la edad en la que se adquieren los diferentes hitos relativos al desarrollo de la independencia en las AVDs, está condicionado por el país de origen, siendo por tanto la cultura un valor determinante en el desarrollo de la autonomía en el niño.

Esto cobra especial relevancia a la hora de evaluar el desarrollo con pruebas creadas en contextos culturales diferentes, debiendo llevarse a cabo, no sólo un proceso de traducción y adaptación cultural, sino también un análisis de la aplicabilidad de los valores normativos de la población origen y población diana.

Comparar resultados de estudios en los que no se tiene constancia de que se han controlado las mismas variables, se acepta que es una limitación importante de este estudio. No obstante, es una práctica relativamente frecuente ya que permite detectar indicios que podrán comprobar posteriores investigaciones. Los resultados presentados señalan diferencias entre distintas poblaciones y en qué sentido van esas diferencias, por lo tanto es necesario diseñar estudios que confirmen si estas diferencias se deben a las diferentes pautas de crianza, a la valoración de los padres de la socialización o la independencia de sus hijos o a otros factores, tales como el momento temporal, las condiciones socioeconómicas de los padres o del país. En definitiva, asegurar un buen control de la muestra y de las variables estudiadas. Señalar por tanto, que la metodología utilizada se presenta como una de las limitaciones de este estudio y que sería necesario un análisis más profundo, utilizando una metodología más cualitativa que permita ahondar en las diferentes cuestiones anteriormente mencionadas.

\section{Conclusiones}

La cultura influye de forma determinante en los patrones de desarrollo en las habilidades relativas a la adquisición de la independencia en las AVDs, como demuestra las diferencias significativas encontradas en las poblaciones española, noruega y taiwanesa. Las intervenciones que desde Terapia Ocupacional se lleven a cabo en el ámbito de la infancia, debería tener en cuenta cuestiones tales como la importancia que los padres dan la independencia de sus hijos, los estilos de crianza propios de cada unidad familiar, así como el entorno físico. Por otro lado, no se puede afirmar que estos estudios se hayan realizado en contextos sociales y culturales absolutamente comparables. Por ello sería conveniente que los estudios transculturales se realizaran por grupos de investigación que aseguren que las muestras, los procedimientos sean homogéneas y que el único factor diferenciador sea la cultura. Igualmente 
sería interesante seguir realizando estudios que permitan comparar el desarrollo de las ocupaciones en diferentes contextos culturales, así como trabajar en la creación de herramientas que tengan ya en cuenta estas cuestiones.

Se señala como una limitación de este estudio el que los datos que se comparan han sido recogidos por tres equipos diferentes, y por tanto no hay garantía de control sobre la muestra y la inexistencia de estudios de investigación que analicen en profundidad las diferencias desde las teorías etno parentales o desde los ambientes de desarrollo, siendo necesaria una metodología más cualitativa que permita profundizar más.

\section{Referencias}

ADAIR, P. M. et al. Familial and cultural perceptions and beliefs of oral hygiene and dietary practices among ethnically and socio-economicall diverse groups. Community Dental Health, London, v. 21, p. 102-111, 2004.

BERG, M. et al. Cross-cultural validation of the pediatric evaluation of disability inventory (PEDI) norms in a randomized Norwegian population. Scandinavian Journal of Occupational Therapy, Oslo, v. 15, n. 3, p. 143$152,2008$.

BERG, M. et al. Reliability of the pediatric evaluation of disability inventory (PEDI). Physical and Occupational Therapy in Pediatrics, London, v. 24, n. 3, p. 61-77, 2004.

CASE-SMITH, J.; CLIFFORD O'BRIEN, J. Occupational Therapy for children. Missouri: Mosby Elsevier, 2010.

CHEN, K. L. et al. Pediatric evaluation of disability inventory: a cross-cultural comparison of daily function between Taiwanese and American children. Research in Developmental Disabilities, New York, v. 31, n. 6, p. 1590-1600, 2010.

CHEN, K. L. et al. Reliability and validity of a Chinese version of the pediatric evaluation of disability inventory in children with cerebral palsy. Journal of Rehabilitation Medicine, Sweden, v. 41, n. 4, p. 273-278, 2009.

CHUA, A. Madre tigre, hijos leones. Madrid: Temas de Hoy, 2011.

CÓRDOBA IÑESTA, A. Teorías del desarrollo. In: CÓRDOBA IÑESTA, A.; DESCALS TOMÁS, A.; GIL LLARIO, A. (Ed.). Psicología del desarrollo en la edad escolar. Madrid: Pirámide, 2008. p. 39-52.

DICHTELMILLER, M. et al. The relationship of parental knowledge to the development of extremely low birth weight infants. Journal of Early Intervention, Reston, v. 16, n. 3, p. 210-220, 1992.

FLORES MARTOS J. Actividades de la vida diaria desde una perspectiva antropológica. In: MORUNO
MIRALLES, P.; ROMERO AYUSO, D. M. (Ed.). Actividades de la vida diaria. Barcelona: Masson, 2006. p. 399-416.

GARCÍA BÁSCONES, M. Adaptación transcultural y versión española de la escala de discapacidad Pediatric evaluation of disability inventory (PEDI). 2013. $134 \mathrm{f}$. Tesis (Doctoral Medicina Física y de Rehabilitación) Universidad Complutense, Madrid, 2013.

GREENFIELD, P. M.; SUZUKI, L. K. Culture and Human developmental: implications for parenting, education, pediatrics and mental health. In: DAMON, W.; SIGEL, I. E.; RENNINGER, K. A. (Ed.). Handbook of child psychology. New York: John Willey, 1998. p. 10591109.

GROSS-LOH, C. Parenting without borders: surprising lessons parents around the world can teach us. New York: Penguin, 2013.

HALEY, S. et al. Pediatric Evaluation of Disability Inventory (PEDI). Development, standardization and manual administration. Boston: Trustees of Boston University, 1992.

IZZEDIN, R.; PACHAJOA, A. Pautas, prácticas y creencias acerca de la crianza...ayer y hoy. Liberabit, Lima, v. 15, n. 2, p. 109-115, 2009.

LAW, M. et al. The Person-Environment-Occupation Model: a transactive approach to occupational performance. Canadian Journal of Occupational Therapy, Ottowa, v. 63, n. 1, p. 9-23, 1996.

MASAGATANI, G. Ambientes humanos y no humanos. In: HOPKINS, H.; SMITH, H. (Ed.). Terapia Ocupacional. Madrid: Panamericana, 1998. p. 145-148.

MATTINGLY, C.; BEER, D. Interpretación de la cultura en un contexto terapeútico. In: HOPKINS, H.; SMITH, H. (Ed.). Terapia Ocupacional. Madrid: Panamericana, 1998. p. 154-161.

PARMAR, P. H.; SUPER, C. S. Asian and Euro-American parents' ethnotheories of play and learning: effects on preschool children's home routines and school behaviour. International Journal of Behavioral Development, London, v. 28, n. 2, p. 97-104, 2004.

RIGBY, P.; LETTS, L. Enviorment and occupational performance: theoretical considerations. In: LETTS, L.; RIGBY, P.; STEWART, D. (Ed.). Using enviorments to enable occupational performance. New Jersey: Thorofare, 2003. p. 17-32.

RIKHY, S. et al. Gauging knowledge of developmental milestones among Albertan adults: a cross-sectional survey. BMC Public Health, London, v. 10, n. 1, p. 1-9, 2010.

SANTAMARÍA VÁZQUEZ, M. Aplicabilidad de la Evaluación pediátrica de la discapacidad (PEDI) en po- 
blación española. Madrid: Universidad Rey Juan Carlos, 2014.

SAVE THE CHILDREN. State of the World's Mothers 2013. Westport, 2013.

SAVE THE CHILDREN. State of the World's Mothers 2014. Westport, 2014.
STEVENS, J. H. Child development knowledge and parenting skills. Family Relations, United States, v. 33, n. 2, p. 237-244, 1984.

WISE, S.; DA SILVA, L. Differential parenting of children from diverse cultural backgrounds attending child care. Sidney: Commonweath of Australia, 2007.

\section{Contribución de los Autores}

Montserrat Santamaría-Vázquez - Análisis de los resultados, redacción del texto. Valeriana Guijo-Blanco Dirección del proyecto, redacción y revisión del texto. Todos los autores aproban la versión final del texto. 